\title{
THE MAXIMUM TERM OF A POWER SERIES
}

\author{
G.S. SRIVASTAVA \\ Department of Mathematics \\ University of Roorkee \\ Roorkee, India \\ and \\ O.P. JUNEJA \\ Department of Mathematics \\ Indian Institute of Technology \\ Kanpur, India \\ (Received March 30, 1984)
}

ABSTRACT. Let $\sum_{n=0}^{\infty} a_{n} z^{\lambda}$ be a power series, representing an analytic function $f(z)$ in the disc $|z|<R$. A characterization for the type of such functions was obtained by the authors [J. Math. Anal. Appl. $81(1981), 1-7$ ] in terms of the maximum term and rank. It is proved in this paper by means of an example, that a similar relation does not hold in general for lower type and sufficient conditions have been obtained for the validity of the corresponding result for lower type. Alternative coefficient characterization for type and lower type have been given and a necessary and sufficient condition for the analytic function $f(z)$ to be of perfectly regular growth has been obtained.

KEY WORDS AND PHRASES. Analytic function, order, type, lower type, maximum term, and rentral, index.

AMS SUR.IECT CLASSIFICATION CODE. $30 B 10$.

1. INTRODUCTION.

Let $f(z)=\sum_{n=0}^{\infty} a_{n} z^{\lambda}$ be an analytic function with radius of convergence $R(0<R<\infty)$. For $0 \leq r<R$, we set $M(r)=\max _{|z|=r}|f(z)|, m(r)=\max \left\{\left|a_{n}\right| r^{\lambda}\right\}$ and $N(r)=\max _{n \geq 0}\left\{\lambda_{n}: m(r)=\left|a_{n}\right| r^{\lambda} n\right.$. Then $M(r), m(r)$, and $N(r)$ are called the maximum modulus, maximum term and central index respectively. The order $\rho$ of $f(z)$ is deined as

$$
\lim _{r \rightarrow R} \sup \frac{\log ^{+} \log ^{+} M(r)}{\log x}=\rho, 0 \leq \rho \leq \infty,
$$

where $x=R r /(R-r)$. When $\rho<\infty, M(r)$ can be replaced by $m(r)$ in $(1,1)[1$, Lemma2]. Further for $0<\rho<\infty$, the type $T$ and lower type $t$ of $f(z)$ are defined as

$$
\lim _{r \rightarrow R} \sup \frac{\log m(r)}{x^{\rho}}=\frac{T}{t}
$$


The function $f(z)$ is said to be of regular growth if limit exists on the left hand side of (1.1). Further, $f(z)$ is said to be of perfectly regular growth if $0<t=$ $T<\infty$. Maclane [2] obtained the following formula for the order $\rho$ of $f(z)$ :

$$
\lim _{n \rightarrow \infty} \sup \frac{\log ^{+} \log ^{+}\left|a_{n}\right|}{\log \lambda_{n}}=\frac{\rho}{\rho+1} \text {. }
$$

Further, Bajpai, Tanne, and Whittier [3] obtained the following coefficient characterization for $\mathrm{T}$ and $t$ :

$$
\lim _{n \rightarrow \infty} \sup _{\inf } \frac{\left[\log ^{+}\left(\left|a_{n}\right| R^{\lambda}\right)\right](\rho+1)}{B \lambda_{n}^{\rho}}=\frac{T}{t}
$$

where $B=(\rho+1)^{\rho+1}(R / \rho)^{\rho}$. The expression for the lower type $t$ holds under the conditions that (i) $\lambda_{n} \simeq \lambda_{n+1}$ and (ii) $\psi(n)=\frac{\log \left|a_{n} / a_{n+1}\right|}{\lambda_{n+1}-\lambda_{n}}$ is a non decreasing function of $\mathrm{n}$.

In a recent paper of [4], we obtained a formula for the type $T$ in terms of $M(r)$ and $N(r)$. Thus we have

$$
\left(\frac{\rho+1}{\rho}\right)^{\rho+1}\left(\frac{\rho T}{R}\right)=\lim _{r \rightarrow R} \sup \frac{N(r)}{x^{\rho+1}}\left\{1+\frac{x}{r} \frac{\log m(r)}{N(r)}\right\}(\rho+1)
$$

In this paper, we show by means of an example that a corresponding result for lower type $t$ does not always hold. We shall obtain the corresponding result for lower type under certain conditions. In the process, we also obtain formulae corresponding to (1.4) without any additional conditions on $\lambda_{n}$ or $a_{n}$ 's, thus improving upon $(1.4)$.

2. WE PROVE THE FOLLOWING;

THEOREM 1. Let $f(z)=\sum_{n=0}^{\infty} a_{n} z^{\lambda}$ be analytic in $|z|<R$ and of order $\rho(0<\rho<\infty)$, type $T$ and lower type $t$. Let $\left\{n_{k}\right\}$ denote the sequence of principal indices of $f$ and $\left\{r_{k}\right\}$ the sequence of jump points of $N(r), 1 . e .$,

$$
N(r)=n_{k} \text { for } r_{k} \leq r<r_{k+1}, k=1,2,3 \ldots
$$

Then

$$
\begin{aligned}
& \lim _{k \rightarrow \infty} \sup \left(\frac{\rho}{R n_{k}}\right)\left[\frac{\log +\left(\left.\right|_{\frac{n_{k}}{\rho+1}} ^{R_{R}}{ }^{n_{k}}\right)}{(\rho+1)}\right. \\
& \lim _{k \rightarrow \infty} \inf \frac{\log m\left(r_{k}\right)}{\left(x_{k}\right)^{\rho}}=t,
\end{aligned}
$$

where $x_{k}=R r_{k} /\left(R-r_{k}\right)$.

Before providing the above theorem, we give an example to show that (1.5) does not always hold for the lower type.

EXAMPLE. Let us consider the function $f(z)={ }_{n=0}^{\infty} e^{2^{n}} z^{4^{n}}$. Then it can be easily seen that $f(z)$ is analytic in $|z|<1$, of order $\rho=1$. Futther, $N(r)=4^{n}$, $m(r)=e^{2^{n}} r^{4^{n}}$ for

$$
\mathrm{e}^{-1 / 3\left(2^{\mathrm{n}-1}\right)} \leq \mathrm{r}<\mathrm{e}^{-1 / 3\left(2^{\mathrm{n}}\right)} .
$$


Hence, from (2.1) and (2.2) above, we have $T=1 / 4, t=2 / 9$. However, in this case (for $R=1$ and $\rho=1$ )

$$
\lim _{r \rightarrow R} \frac{N(r)}{x^{\rho+1}}\left\lceil 1+\frac{x}{r} \frac{\log m(r)}{N(r)}\right]^{\rho+1}=1>\frac{8}{9}=\left(\frac{\rho+1}{\rho}\right)^{\rho+1}\left(\frac{\rho t}{R}\right) .
$$

Hence we see that although limit exists on the right hand side of (1.5), the equality fails to hold for lower type $t$. The above inequality can be generalized as follows: THEOREM 2. Let $f(z)$ be defined as before. Then

$$
\left(\frac{\rho+1}{\rho}\right)^{\rho+1}\left(\frac{\rho t}{R}\right) \leq \lim _{r \rightarrow R} \inf \frac{N(r)}{x^{\rho+1}}\left[1+\frac{x}{r} \frac{\log m(r)}{N(r)}\right]^{(\rho+1)} .
$$

In order to get reverse inequality for $t$, we prove THEOREM 3. Let $f(z)$ be defined as in Theorem 1. Then for any increasing sequence $\left\{r_{n}\right\}$ tending to $R$, we have

$$
\left(\frac{\rho+1}{\rho}\right)^{\rho+1}\left(\frac{\rho t}{R}\right) \geq \lim _{n \rightarrow \infty} \text { inf } \frac{N\left(r_{n-1}\right)}{\left(x_{n}\right)^{\rho+1}}\left[1+\frac{x_{n}}{r_{n}} \frac{\log m\left(r_{n}\right)}{N\left(r_{n}\right)}\right]^{\rho+1},
$$

where $x_{n}=R \quad r_{n} /\left(R-r_{n}\right)$.

The following corollaries are immediate consequences of Theorem 3 . COROLLARY 1. We have

$$
\left(\frac{\rho+1}{\rho}\right)^{\rho+1}\left(\frac{\rho t}{R}\right) \geq \max _{\left\{r_{n}\right\}} \lim _{n \rightarrow \infty} \inf \frac{N\left(r_{n-1}\right)}{x_{n}^{\rho+1}}\left\{1+\frac{x_{n}}{r_{n}} \frac{\log m\left(r_{n}\right)}{N\left(r_{n}\right)}\right\}^{\rho+1},
$$

where the maximum on the right hand side is taken over all increasing sequences $\left\{r_{n}\right\}$ such that $r_{n} \rightarrow R$ as $n \rightarrow \infty$.

COROLLARY 2. We have

$$
\begin{aligned}
& \left(\frac{\rho+1}{\rho}\right)^{\rho+1}\left(\frac{\rho t}{R}\right) \geq\left\{\max _{\left\{r_{n}\right\}} \lim _{n \rightarrow \infty} \inf \frac{N\left(r_{n-1}\right)}{N\left(r_{n}\right)}\right\} \\
& \left\{\lim _{r \rightarrow R} \inf \frac{N(r)}{x^{\rho+1}}\left[1+\frac{x}{r} \frac{\log m(r)}{N(r)}\right]^{\rho+1}\right\},
\end{aligned}
$$

where $x=R r /(r-r)$ and $\left\{r_{n}\right\}$ is as defined above.

Lastly we state two theorems.

THEOREM 4. If $f(z)$ satisfies the condition of Theorem 1 and there exists an increasing sequence of positive real numbers $\left\{r_{n}\right\}$ such that $r_{n} \rightarrow R$ as $n \rightarrow \infty$ and

$$
N\left(r_{n-1}\right) \simeq N\left(r_{n}\right) \text { as } n \rightarrow \infty \text {, }
$$

then

$$
\left(\frac{\rho+1}{\rho}\right)^{\rho+1}\left(\frac{\rho t}{R}\right)=\lim _{r \rightarrow R} \inf \frac{N(r)}{x^{\rho+1}}\left[1+\frac{x}{r} \frac{\log m(r)}{N(r)}\right]^{\rho+1} .
$$

THEOREM 5. If $f(z)$ satisfies the conditions of Theorem 1 and is of perfectly regular growth, then

$$
\lim _{r \rightarrow R} \frac{N(r)}{x^{\rho+1}}\left[1+\frac{x}{r} \frac{\log m(r)}{N(r)}\right]^{\rho+1}
$$

exists.

Conversely, if limit in (2.9) exists and equals $A$ and $N(r)$ satisfies the con- 
dition (2.7), then $f(z)$ is of perfectly regular growth and

$$
t=T=\left(\frac{\rho}{\rho+1}\right)^{\rho+1}\left(\frac{A R}{\rho}\right)
$$

3. PROOFS OF THEOREMS.
THEOREM 1. Let us set $P(r)=\frac{\log m(r)}{[R r /(R-r)]^{\rho}}$. Then $P(r)=\frac{\log \left|a_{n_{k}}\right|+n_{k} \log r}{[R r /(R-r)]^{\rho}}$ for $r_{k} \leq r<r_{k+1}$. It is clear that $P(r)$ is a differentiable function of $r$ for $\mathrm{r}_{\mathrm{k}} \leq \mathrm{r}<\mathrm{r}_{\mathrm{k}+1}$. Differentiating, we have

$$
P^{\prime}(r)=\left(\frac{R}{R-r}\right)^{1-\rho} r^{1-\rho} Q_{k}(r)
$$

where $Q_{k}(r)=\left(\frac{R-r}{R}\right) n_{k}-\rho\left(\log \left|a_{n_{k}}\right|+n_{k} \log r\right)$. Since $\frac{R-r}{R} \simeq \log (R / r)$ as $r \rightarrow R$, it can be easily seen that $Q_{k}(r)=0$ for

$$
r=r_{k}^{*} \simeq R^{1 /(\rho+1)}\left|a_{n_{k}}\right|^{-\rho /(\rho+1) n_{k}}
$$

Further, for fixed $k$, the function $P(r)$ has an absoute maxima for $r=r_{k}^{*}$ only. If $r_{k}<r_{k}^{*}<r_{k+l}$, then the function $P(r)$ increases for $r_{k} \leq r \leq r_{k}^{*}$. In case $r_{k}^{*} r_{k}, P(r)$ decreases for $r_{k} \leq r \leq r_{k+1}$, while if $r_{k}^{*} \geq r_{k+1}$, then $P(r)$ is a non decreasing function of $r$ for $r_{k} \leq r \leq r_{k+1}$. In every case, we have for $r_{k} \leq r \leq r_{k+1}$

$$
\begin{aligned}
& P(r) \geq P\left(r_{k}\right), \text { or } \\
& P(r) \geq P\left(r_{k+1}\right) .
\end{aligned}
$$

Thus (3.3) and (3.4) give

$$
\lim _{r \rightarrow R} \inf P(r) \geq \lim _{k \rightarrow \infty} \inf P\left(r_{k}\right),
$$

But we obviously have

$$
\lim _{r \rightarrow R} \text { inf } P(r)=\lim _{k \rightarrow \infty} \text { inf } P\left(r_{k}\right)
$$

Thus (3.5) and (3.6) lead to (2.2).

To prove (2.1), we note that for $r_{k} \leq r<r_{k+1}$,

$$
P(r) \leq \frac{\log \left|a_{n_{k}}\right|+n_{k} \log r_{k}^{*}}{\left[R r_{k}^{*} /\left(R-r_{k}^{*}\right)\right]^{0}}
$$

since for fixed $k, P(r)$ has an absolute maxima at $r=r_{k}^{*}$. The above inequality, after simple calculation leads to

$$
\begin{aligned}
T & =\lim _{r \rightarrow R} \sup P(r) \\
& \left.\leq \lim _{k \rightarrow \infty} \sup \left(\frac{\rho}{R n_{k}}\right) \rho \frac{\log \left(\left|a_{n_{k}}\right| R^{n_{k}}\right) \rho+1}{\rho+1}\right]
\end{aligned}
$$

The reverse inequality is trivial since

$$
T=\lim _{r \rightarrow R} \sup P(r) \geq \lim _{k \rightarrow \infty} \sup P\left(r_{k}^{*}\right)
$$




$$
=\lim _{k \rightarrow \infty} \sup \left(\frac{\rho}{R n_{k}}\right)^{\rho}\left[\frac{\log \left(\left|a_{n_{k}}\right| R^{n_{k}}\right)}{\rho+1}\right]^{\rho+1} \text {. }
$$

Thus we get (2.1) on combining (3.7) and (3.8). This proves Theorem 1 .

REMARK. The result $(2.1)$ is an improvement over the corresponding result (1.4) of Bajpai et.al. [3].

THEOREM 2. It is easy to verify that for $\rho>0$ and $u \geq 0$,

$$
\rho u \leq\left(\frac{\rho}{\rho+1}\right)^{\rho+1}(1+u)^{\rho+1} \text {. }
$$

The inequality (2.3) now follows on making proper substitutions for $u$ and proceeding to limits as $\mathbf{r} \rightarrow \mathrm{R}$.

THEOREM 3. To prove $(2.4)$, we put

$$
\lim _{n \rightarrow \infty} \inf \frac{N\left(r_{n-1}\right)}{x_{n}^{p+1}}\left[1+\frac{x_{n}}{r_{n}} \frac{\log m\left(r_{n}\right)}{N\left(r_{n}\right)}\right]^{p+1}=q .
$$

Then for $0<\epsilon<q$, we have for $n>n_{0}(\epsilon)$,

$$
\frac{N\left(r_{n-1}\right)}{r_{n}^{\rho+1}}\left[\frac{R-r_{n}}{R}+\frac{\log m\left(r_{n}\right)}{N\left(r_{n}\right)}\right]^{\rho+1}>q-\epsilon .
$$

This leads to

$$
\log m\left(r_{n}\right)>\left[r_{n}\left\{\frac{q-\epsilon}{N\left(r_{n-1}\right)}\right\}^{1 /(p+1)}-\frac{R-r_{n}}{R}\right] N\left(r_{n}\right) .
$$

From the relation $[5, \mathrm{p} .196]$

$$
\log M(r)=\log m\left(r_{0}\right)+\int_{r_{0}}^{r} \frac{N(t)}{t} d t, 0<r_{0} \leq r<R,
$$

we have

$$
\begin{aligned}
\log m(r) & =\log m\left(r_{n}\right)+f_{n}^{r} \frac{N(t)}{t} d t \\
& \geq \log m\left(r_{n}\right)+N\left(r_{n}\right) \log \left(r / r_{n}\right) \\
& =\log m\left(r_{n}\right)+N\left(r_{n}\right)\left\{\log \left(R / r_{n}\right)-\log (R / r)\right\}
\end{aligned}
$$

Using (3.9) and the fact that $\frac{R-r}{R} \simeq \log \left(\frac{R}{r}\right)$ as $r \rightarrow R$, we get

$$
\log m(r)>N\left(r_{n}\right)\left[r_{n}\left\{\frac{q-\epsilon}{N\left(r_{n-1}\right)}\right\}^{1 /(p+1)}-\frac{R-r}{R}\right] .
$$

Let $\left\{s_{n}\right\}$ be a sequence defined by

$$
s_{n}=R \exp \left[-\frac{p}{p+1}\left\{\frac{R(q-\epsilon)}{N\left(r_{n-1}\right)}\right\}^{1 /(p+1)}\right], n>n_{0} \text {. }
$$

It is clear that $s_{n} \rightarrow R$ as $n \rightarrow \infty$. If $r$ satisfies $s_{n} \leq r<s_{n+1}$, then for $x=\operatorname{Rr} /(R-r)$,

$$
\begin{aligned}
\frac{\log m(r)}{x^{\rho}} & >N\left(r_{n}\right)\left[\frac{R-s_{n+1}}{R s_{n+1}}\right]^{\rho}\left[\frac{(\rho+1) r_{n} \log \left(R / s_{n}\right)}{\rho R}-\frac{R-s_{n}}{R}\right] \\
& \simeq N\left(r_{n}\right)\left[\frac{R-s_{n+1}}{R s_{n+1}}\right]\left[\frac{(\rho+1) r_{n}\left(R-s_{n}\right)}{\rho R^{2}}-\frac{R-s_{n}}{R}\right]
\end{aligned}
$$




$$
\begin{aligned}
& \simeq\left(\frac{\rho}{\rho+1}\right)^{\rho+1} \frac{R(q-\epsilon)\left(R-s_{n}\right)}{\rho\left(R-s_{n+1}\right)} \\
& >\left(\frac{\rho}{\rho+1}\right)^{\rho+1} \frac{(q-\epsilon) R}{\rho} .
\end{aligned}
$$

Proceeding to limits as $r \rightarrow R$, we get

$$
\left(\frac{\rho}{\rho+1}\right)^{\rho+1}\left(\frac{\rho t}{R}\right) \geq q
$$

which yields (2.4). The inequality obviously holds if $q=0$. This proves Theorem 3 .

As already mentioned, Corollaries 1 and 2 are direct consequences of Theorem 3 . Theorem 4 follows on combining (2.3) with (2.6) under the condition (2.7). Hence we omit the proofs.

Lastly we prove

THEOREM 5. Let us suppose that $f(z)$ is of perfectly regular growth, i.e. T $=t$. Then from (1.5) and (2.3), we have

$$
\begin{gathered}
\lim _{r \rightarrow R} \sup \frac{N(r)}{x^{\rho+1}}\left[1+\frac{x}{r} \frac{\log m(r)}{N(r)}\right]^{\rho+1}=\left(\frac{\rho+1}{\rho}\right)^{\rho+1}\left(\frac{\rho t}{R}\right) \\
\leq \lim _{r \rightarrow R} \inf \frac{N(r)}{x^{\rho+1}}\left[1+\frac{x}{r} \frac{\log m(r)}{N(r)}\right]^{\rho+1},
\end{gathered}
$$

that is, limit exists in (2.9).

Conversely, let

$$
\lim _{r \rightarrow R} \frac{N(r)}{x^{\rho+1}}\left[1+\frac{x}{r} \frac{\log m(r)}{N(r)}\right]^{\rho+1}=A
$$

and suppose that (2.7) is satisfied for some sequence $\left\{r_{n}\right\}$. Then from (2.8) and $(1.5)$, we have

$$
\left(\frac{\rho+1}{\rho}\right)^{\rho+1}\left(\frac{\rho t}{R}\right)=\left(\frac{\rho+1}{\rho}\right)^{\rho+1}\left(\frac{\rho t}{R}\right)=A \text {, }
$$

i.e. $T=t=\left(\frac{\rho+1}{\rho}\right)^{\rho+1}\left(\frac{A R}{\rho}\right)$.

This proves Theorem 5 .

\section{REFERENCES}

1. SONS, L.R. Regularity of growth and gaps, J. Math. Anal. Appl. 24 (2) (1968), 296306.

2. MacLANE, G.R. Asymptotic values of holomorphic functions, Rice University Studies, Houston, (Texas), 1963.

3. BAJPAI, S.K., TANNE, J., and WHITTIER, D. A decompostion theorem for an analytic function, J. Math. Ana1. App1. 48(1974), 736-743.

4. SRIVASTAVA, G.S. and JUNEJA, O.P. The maximum term of a power series, J. Math. Ana1. App1. 81(1) (1981), 1-7.

5. VALIRON, G. Fonction Analytiques, Press Universitaires de France, Paris, 1964. 


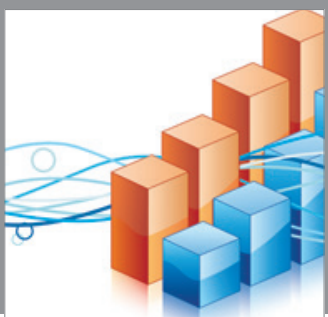

Advances in

Operations Research

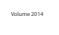

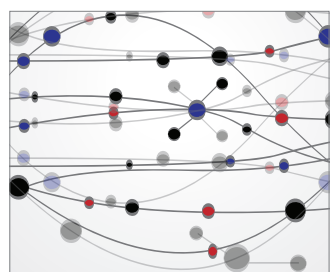

\section{The Scientific} World Journal
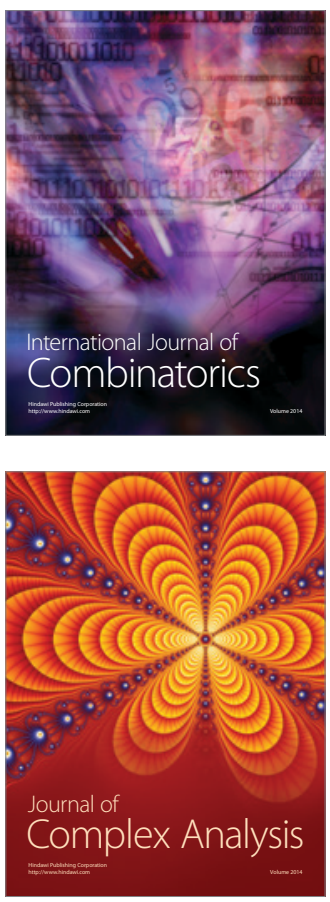

International Journal of

Mathematics and

Mathematical

Sciences
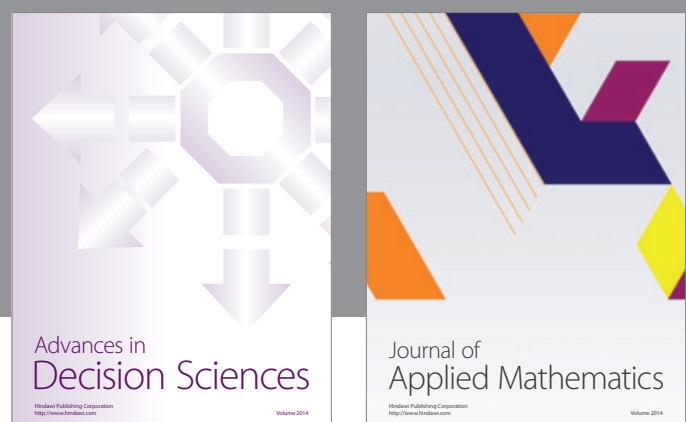

Journal of

Applied Mathematics
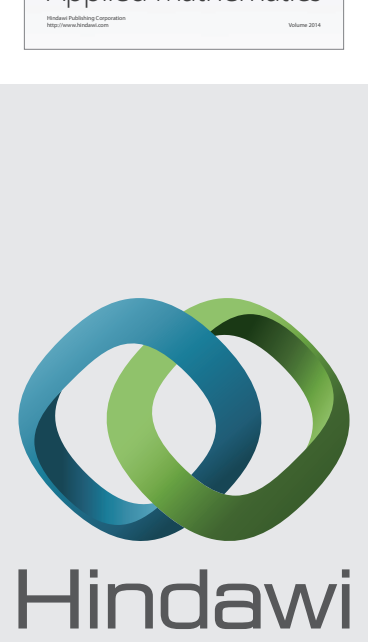

Submit your manuscripts at http://www.hindawi.com
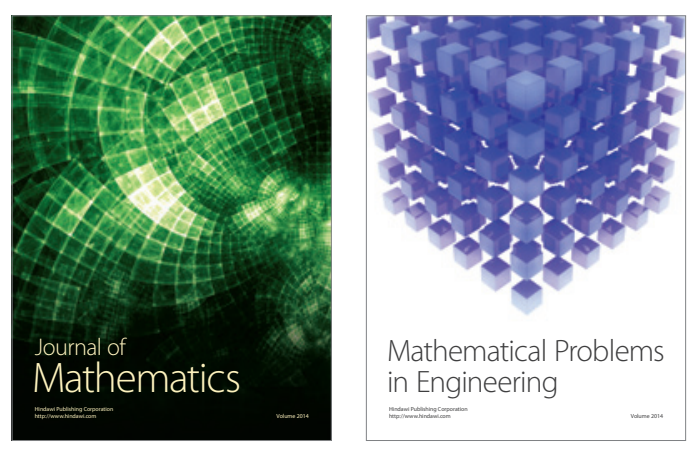

Mathematical Problems in Engineering
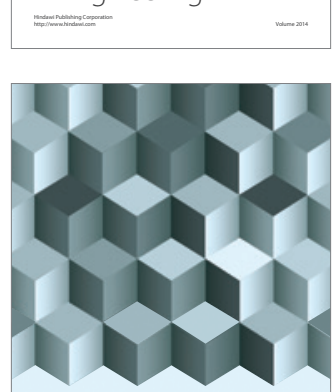

Journal of

Function Spaces
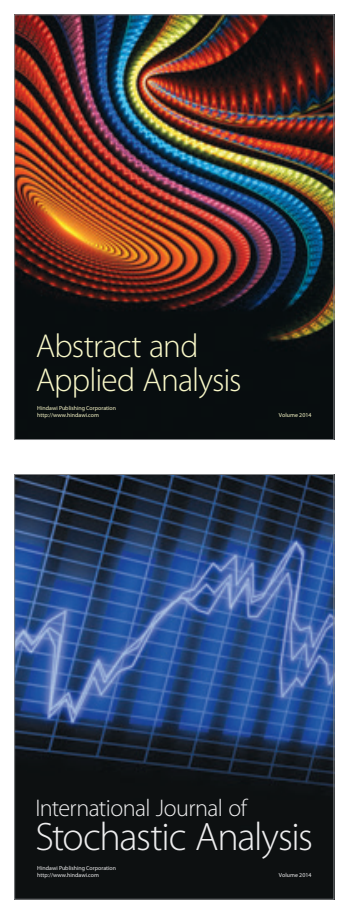

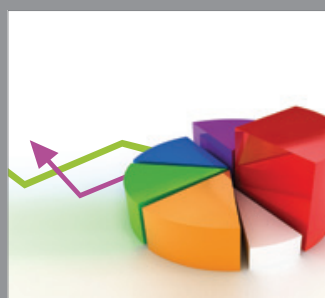

ournal of

Probability and Statistics

Promensencen
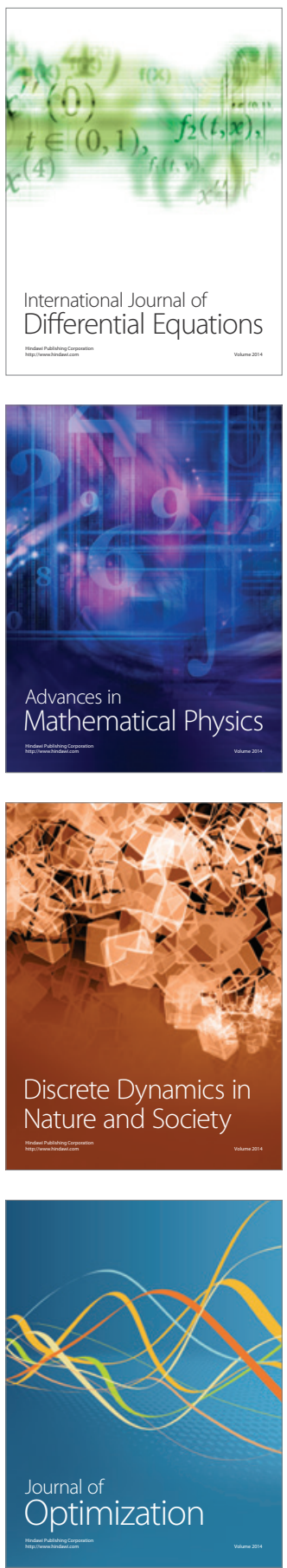